\title{
Electromagnetic Wave Absorber with Isotropic and Anisotropic Metamaterials
}

\author{
Dong Yunxia \\ School of Electrical and Electronic Engineering, North China Electric Power University, Beijing, China
}

\section{Email address:}

dyx2007@ncepu.edu.cn

\section{To cite this article:}

Dong Yunxia. Electromagnetic Wave Absorber with Isotropic and Anisotropic Metamaterials. International Journal of Materials Science and Applications. Vol. 6, No. 6, 2017, pp. 302-308. doi: 10.11648/j.ijmsa.20170606.16

Received: November 10, 2017; Accepted: November 22, 2017; Published: December 6, 2017

\begin{abstract}
The absorption characteristics of the electromagnetic wave propagating through one dimensional system with the metamaterials are studied basing on the transfer matrix method. The relations between the absorptivity with the frequency of the incident wave and the thickness of the metamaterials are calculated. The results show that the absorptivity is about $100 \%$ near the resonant frequency of the isotropic metamaterials. This means that the metamaterials can be used as a narrow band absorber. The thickness of the metamaterials determines the width of the band. Furthermore the absorption characteristics of the multilayers system with the isotropic and anisotropic metamaterials are studied. The band width increases with the addition of the layer number. The absorption is dependent on the polarization direction of the incident electromagnetic wave for the anisotropic metamaterials. Thus the anisotropic metamaterials can be used to be the polarization tunable absorber. The layer number can also determine the frequency for the maximum absorption as for the multilayer system.
\end{abstract}

Keywords: Absorber, Metamaterials, Multilayer System, Polarization

\section{Introduction}

The metamaterials have attracted a great deal of attention from both theoretical and experimental sides in recent several decades. The metamaterials have the artificial structure and the electromagnetic parameters are dependent on the resonant of the electric and the magnetic field. In some frequency region the effective permittivity and the permeability of the materials can be simultaneously negative due to the periodically positioned scattering elements. The materials are also called as the left-handed materials because the electric field, the magnetic field and the wave vector form a left-handed relation in the materials. These media exhibit a number of unusual electromagnetic properties such as negative refractive index [1] amplification of evanescent wave [2], subwavelength cavity resonator [3], zero averaged refractive index band gap [4] etc. Due to that the unit resonance structures of metamaterials are usually anisotropic [1], people became interested in the anisotropic properties and revealed many intriguing phenomena in different kinds of anisotropic metamaterials [5-9].

Compared with the isotropic materials, anisotropic materials have more freedom to control the propagation and polarization of the electromagnetic waves. People have shown that the property of negative refraction is not confined to materials with negative definite permittivity tensor and permeability tensor, but can be expected to occure in certain classes of uniaxially anisotropic media. Research has shown that electromagnetic wave polarizations can be manipulated freely through reflections by anisotropic metamaterials [9, 10]. A single metamaterial plate can serve as a bandpass filter, transparent wall, and polarization converter under illumination from differently polarized waves [11]. A study of the quantization of the electromagnetic field in anisotropic metamaterials has also been performed; the input-output relations of quantized radiation have been derived for a single anisotropic metamaterial plate $[12,13]$. Some unusual properties for the polarized photon transmitting through a three layer cavity containing anisotropic metamaterials are studies in Ref. [14].

Since most proposed metamaterials are metallic resonant structures and rely on strong resonances, the absorption losses are inevitable. The loss of the metamaterials often degrades their performance. However, the loss becomes useful and it could be significantly enhanced in perfect absorber. Since its first presentation [15], metamaterial 
absorbers have received considerable attention and many absorbers have been proposed [16-18]. An effective method to broaden the absorption bandwidth is to make the metamaterial units resonate at several neighboring frequencies. Following this design strategy, polarization insensitive or polarization dependent broadband absorbers have been widely demonstrated from microwave to optical. Huang et al. [19] presented the polarization dependent broadband absorber based on three I-shaped resonators. Grant et al. [20] obtained polarization insensitive wideband absorbers by stacking multiple patterns.

But the research about the electromagnetic wave propagating through a multilayer cavity is not studied at present. It is meaningful to study the absorption characteristics of the wave propagation through multilayer structures of anisotropic metamaterials. In this work, we are interested in the optical anisotropy and the transmission properties at a planar multilayer cavity with a negative permittivity, whose wave characteristics have yet to be examined. In this paper, a polarization tunable absorber formed by anisotropic metamaterials is presented. The absorber frequency is able to be tuned by merely changing the polarization of the light. The tunable mechanism originates from the variation of the effective length of the absorber. This feature makes the proposed structure very useful in manipulating polarization of light and detecting waves with specific polarization.

\section{Theoritcal Derivation and Calculations}

It is a normal method to calculate the transfer of the electromagnetic in one dimensional multilayer structure to use the transfer matrix method (TMM). For a one dimensional $N$ layer structure, the relative permittivity tensor $\overrightarrow{\mathcal{E}}$ and relative permeability tensor $\vec{\mu}$ of the anisotropic medium in each layer are given by

$$
\vec{\varepsilon}=\left(\begin{array}{ccc}
\varepsilon_{x} & 0 & 0 \\
0 & \varepsilon_{y} & 0 \\
0 & 0 & \varepsilon_{z}
\end{array}\right)
$$

and

$$
\vec{\mu}=\left(\begin{array}{ccc}
\mu_{x} & 0 & 0 \\
0 & \mu_{y} & 0 \\
0 & 0 & \mu_{z}
\end{array}\right)
$$

For isotropic metarial, the diagonal elements in the above matrix are the same. From the Maxwell equations we can get

$$
\vec{k} \times\left[\vec{\mu}^{-1}(\vec{k} \times \vec{E})\right]+\frac{\omega^{2}}{c^{2}} \vec{\varepsilon} \cdot \vec{E}=0
$$

Here $\vec{k}$ is the wave vector of the electromagnetic wave

$$
\vec{E}(\vec{r}, t)=\sum_{\sigma=1}^{4} E_{\sigma} \hat{e}_{\sigma} e^{i\left[k_{x} x+k_{y} y+k_{z \sigma} z-\omega t\right]}
$$

with $E_{\sigma}$ as the expansion coeiffecients, and $\hat{e}_{\sigma}$ as the vector of the electric field.

$$
\hat{e}_{\sigma}=\left(X_{\sigma}^{2}+Y_{\sigma}^{2}+Z_{\sigma}^{2}\right)^{-1 / 2}\left(X_{\sigma} \hat{x}+Y_{\sigma} \hat{y}+Z_{\sigma} \hat{z}\right)
$$

The parameters in the above equation are defined as

$$
\begin{aligned}
& X_{\sigma}=\left[(\omega / c)^{2} \varepsilon_{y}-\mu_{z}^{-1} k_{x}^{2}-\mu_{x}^{-1} k_{z}^{2}\right]\left[(\omega / c)^{2} \varepsilon_{z}-\mu_{y}^{-1} k_{x}^{2}-\mu_{x}^{-1} k_{y}^{2}\right]-\mu_{x}^{-2} k_{y}^{2} k_{z}^{2} \\
& Y_{\sigma}=\mu_{x}^{-1} \mu_{y}^{-1} k_{x} k_{y} k_{z}^{2}-\mu_{z}^{-1} k_{x} k_{y}\left[(\omega / c)^{2} \varepsilon_{z}-\mu_{y}^{-1} k_{x}^{2}-\mu_{x}^{-1} k_{y}^{2}\right] \\
& Z_{\sigma}=\mu_{x}^{-1} \mu_{z}^{-1} k_{x} k_{y}^{2} k_{z}-\mu_{y}^{-1} k_{x} k_{z}\left[(\omega / c)^{2} \varepsilon_{y}-\mu_{z}^{-1} k_{x}^{2}-\mu_{x}^{-1} k_{z}^{2}\right]
\end{aligned}
$$

We can also get the magnetic field from the Maxwell equations as

$$
\vec{H}(\vec{r}, t)=\sum_{\sigma=1}^{4} E_{\sigma} \hat{h}_{\sigma} e^{i\left[k_{x} x+k_{y} y+k_{z \sigma} z-\omega t\right]}
$$

with

$$
\hat{h}_{\sigma}=\frac{1}{\mu_{0} \omega} \vec{\mu}^{-1} \cdot\left(\vec{k} \times \hat{e}_{\sigma}\right)
$$

Here $\mu_{0}$ is the permeability of the vacuum.

The transfer matrix can be obtained by the boundary condition of the electromagnetic field. For the one dimensional multilayer structure, the electromagnetic wave in the $n$-th layer can be expressed as

$$
\left(\begin{array}{l}
\vec{E}^{(n)} \\
\vec{H}^{(n)}
\end{array}\right)=\sum_{\sigma=1}^{4} E_{\sigma}^{(n)}\left(\begin{array}{l}
\hat{e}_{\sigma}^{(n)} \\
\hat{h}_{\sigma}^{(n)}
\end{array}\right) e^{i\left[k_{x} x+k_{y} y+k_{z \sigma}^{(n)}\left(z-z_{n-1}\right)-\omega t\right]}
$$

with $\left\{k_{z 1}, k_{z 2}=-k_{z 1}, k_{z 3}, k_{z 4}=-k_{z 3}\right\}$ as the undetermined coefficients, $\hat{e}_{\sigma}, \hat{h}_{\sigma}^{(n)}$ and $k_{z \sigma}^{(n)}$ are the eigen vector of the electric filed, the magnetic field and the wave vector in the $n$-th layer. The value $z_{\mathrm{n}-1}$ is the start value of coordinate of the $n$-th layer. Using the boundary condition, which is that the tangential component of the electric filed and the magnetic field are equal in $z=z_{\mathrm{n}-1}$, we get

$$
\sum_{\sigma=1}^{4} E_{\sigma}^{(n-1)} \hat{e}_{\sigma}^{(n-1)} \cdot \hat{y}=\sum_{\sigma=1}^{4} E_{\sigma}^{(n)} \hat{e}_{\sigma}^{(n)} \cdot \hat{y} e^{-i k_{z \sigma}^{(n)} d_{n}}
$$

Here $d_{\mathrm{n}}=z_{\mathrm{n}}-z_{\mathrm{n}-1}$ is the thickness of the $n$-th layer material. The above equation can also be written in a matrix format as 


$$
\left(\begin{array}{l}
E_{1}^{(n-1)} \\
E_{2}^{(n-1)} \\
E_{3}^{(n-1)} \\
E_{4}^{(n-1)}
\end{array}\right)=D_{n-1}^{-1} D_{n} P_{n}\left(\begin{array}{l}
E_{1}^{(n)} \\
E_{2}^{(n)} \\
E_{3}^{(n)} \\
E_{4}^{(n)}
\end{array}\right)
$$

with

$$
D_{n}=\left(\begin{array}{llll}
\hat{e}_{1}^{(n)} \cdot \hat{y} & \hat{e}_{2}^{(n)} \cdot \hat{y} & \hat{e}_{3}^{(n)} \cdot \hat{y} & \hat{e}_{4}^{(n)} \cdot \hat{y} \\
\hat{h}_{1}^{(n)} \cdot \hat{x} & \hat{h}_{2}^{(n)} \cdot \hat{x} & \hat{h}_{3}^{(n)} \cdot \hat{x} & \hat{h}_{4}^{(n)} \cdot \hat{x} \\
\hat{h}_{1}^{(n)} \cdot \hat{y} & \hat{h}_{2}^{(n)} \cdot \hat{y} & \hat{h}_{3}^{(n)} \cdot \hat{y} & \hat{h}_{4}^{(n)} \cdot \hat{y} \\
\hat{e}_{1}^{(n)} \cdot \hat{x} & \hat{e}_{2}^{(n)} \cdot \hat{x} & \hat{e}_{3}^{(n)} \cdot \hat{x} & \hat{e}_{4}^{(n)} \cdot \hat{x}
\end{array}\right)
$$

and

$$
P_{n}=\left(\begin{array}{cccc}
e^{-i k_{z 1}^{(n)} d_{n}} & 0 & 0 & 0 \\
0 & e^{-i k_{z 2}^{(n)} d_{n}} & 0 & 0 \\
0 & 0 & e^{-i k_{z 3}^{(n)} d_{n}} & 0 \\
0 & 0 & 0 & e^{-i k_{z 4}^{(n)} d_{n}}
\end{array}\right)
$$

At last, the transfer matrix can be obtained as

$$
\left(\begin{array}{l}
E_{1}^{(0)} \\
E_{2}^{(0)} \\
E_{3}^{(0)} \\
E_{4}^{(0)}
\end{array}\right)=D_{0}^{-1} D_{1} P_{1} D_{1}^{-1} D_{2} P_{2} D_{2}^{-1} \cdots D_{n} P_{n} D_{n}^{-1} D_{n+1}\left(\begin{array}{l}
E_{1}^{(n+1)} \\
E_{2}^{(n+1)} \\
E_{3}^{(n+1)} \\
E_{4}^{(n+1)}
\end{array}\right)=M\left(\begin{array}{l}
E_{1}^{(n+1)} \\
E_{2}^{(n+1)} \\
E_{3}^{(n+1)} \\
E_{4}^{(n+1)}
\end{array}\right)
$$

Here $4 \times 4$ transfer matrix $M$ is defined as

$$
M=D_{0}^{-1} D_{1} P_{1} D_{1}^{-1} D_{2} P_{2} D_{2}^{-1} \cdots D_{n} P_{n} D_{n}^{-1} D_{n+1}
$$

We use $A_{s}, A_{p}, B_{s}, B_{p}$ and $C_{s}, C_{p}$ to represent the incident, reflection and the transmitted wave of the electric field amplitude. Consider the electromagnetic wave is incident from the first layer for the left side, then we have

$$
\left(\begin{array}{l}
A_{s} \\
B_{s} \\
A_{p} \\
B_{p}
\end{array}\right)=M\left(\begin{array}{c}
C_{s} \\
0 \\
C_{p} \\
0
\end{array}\right)=\left(\begin{array}{llll}
M_{11} & M_{12} & M_{13} & M_{14} \\
M_{21} & M_{22} & M_{23} & M_{24} \\
M_{31} & M_{32} & M_{33} & M_{34} \\
M_{41} & M_{42} & M_{43} & M_{44}
\end{array}\right)\left(\begin{array}{c}
C_{s} \\
0 \\
C_{p} \\
0
\end{array}\right)
$$

The reflection and transmission coefficients are then expressed in terms of the elements of the propagation matrix by matching the tangential field components at the external boundaries. When the transfer matrix is block diagonal, the reflection and transmission matrices become diagonal, meaning that the two modes are the TE and TM modes. If the incident wave is $\mathrm{s} / \mathrm{p}$ polarization, the reflection coefficients $\mathrm{r}_{\mathrm{ss}}$ $/ \mathrm{r}_{\mathrm{pp}}$ and $\mathrm{r}_{\mathrm{sp}} / \mathrm{r}_{\mathrm{ps}}$ are respectively

$$
r_{s s}=\left(\frac{B_{s}}{A_{s}}\right)_{A_{p}=0}=\frac{M_{21} M_{33}-M_{23} M_{31}}{M_{11} M_{33}-M_{13} M_{31}}
$$

$$
\begin{aligned}
& r_{s p}=\left(\frac{B_{p}}{A_{s}}\right)_{A_{p}=0}=\frac{M_{41} M_{33}-M_{43} M_{31}}{M_{11} M_{33}-M_{13} M_{31}} \\
& r_{p p}=\left(\frac{B_{p}}{A_{p}}\right)_{A_{s}=0}=\frac{M_{11} M_{43}-M_{13} M_{41}}{M_{11} M_{33}-M_{13} M_{31}}
\end{aligned}
$$

$$
r_{p s}=\left(\frac{B_{s}}{A_{p}}\right)_{A_{s}=0}=\frac{M_{11} M_{23}-M_{13} M_{21}}{M_{11} M_{33}-M_{13} M_{31}}
$$

The transmission coefficients for different polarizations are respectively

$$
\begin{gathered}
t_{s s}=\left(\frac{C_{s}}{A_{s}}\right)_{A_{p}=0}=\frac{M_{33}}{M_{11} M_{33}-M_{13} M_{31}} \\
t_{s p}=\left(\frac{C_{p}}{A_{s}}\right)_{A_{p}=0}=\frac{-M_{31}}{M_{11} M_{33}-M_{13} M_{31}} \\
t_{p p}=\left(\frac{C_{p}}{A_{p}}\right)_{A_{s}=0}=\frac{M_{11}}{M_{11} M_{33}-M_{13} M_{31}} \\
t_{p s}=\left(\frac{C_{s}}{A_{p}}\right)_{A_{s}=0}=\frac{-M_{13}}{M_{11} M_{33}-M_{13} M_{31}}
\end{gathered}
$$

According the definition of the absorption, the absorption $\mathrm{A}_{\mathrm{s}}$ for the incident wave which is s polarization is

$$
A_{s}=1-\left|r_{s s}\right|^{2}-\left|r_{s p}\right|^{2}-\left|t_{s s}\right|^{2}-\left|t_{s p}\right|^{2}
$$

The absorption $A_{p}$ for the incident wave which is $p$ polarization can be obtained by

$$
A_{p}=1-\left|r_{p p}\right|^{2}-\left|r_{p s}\right|^{2}-\left|t_{p p}\right|^{2}-\left|t_{p s}\right|^{2}
$$

\section{The Calculation Results}

First we consider a single slab of isotropic metamaterials with the thickness $d$ embedded in the dielectric background (air). Assuming the electromagnetic wave which is linearly polarized along $\mathrm{x}$-direction and $\mathrm{y}$-direction travels along the $\mathrm{z}$ direction. We take the values of the permittivity and permeability as

$$
\begin{gathered}
\varepsilon_{r}(\omega)=\varepsilon(\infty)+\frac{\omega_{p}^{2}}{\omega_{0}^{2}-\omega^{2}-i \gamma_{e} \omega} \\
\mu_{r}(\omega)=\mu(\infty)+\frac{\omega_{p, m}^{2}}{\omega_{0, m}^{2}-\omega^{2}-i \gamma_{m} \omega}
\end{gathered}
$$

for the isotropic metamaterial. Here $\omega_{p}, \omega_{p, m}$ are the plasma 
frequency. $\gamma_{e}, \gamma_{m}$ are the dissipation frequency. $\varepsilon(\infty), \mu(\infty)$ are the static permittivity and permeability. $\varepsilon_{r}(\omega)=\mu_{r}(\omega)$ are used to make the impedance matching. $\omega_{p}=\omega_{p, m}=2 \pi \times 1.25 \mathrm{THz} \quad, \quad \omega_{0}=\omega_{0, m}=2 \pi \times 1.0 \mathrm{THz}$, $\gamma=\gamma_{m}=2 \pi \times 0.1 \mathrm{THz}$ and $\varepsilon(\infty)=\mu(\infty)=1.0$ are used in the calculation.

The calculated result for the relation between the real part and the imaginary part of the relative effective permittivity and the peameability with the frequency is shown in Figure 1. The results show that the real part of the relative effective permittivity changes from the positive value to the negative value when the frequency is $1 \mathrm{THz}$. At the same time the imaginary part has the maximum value and the relative effective permittivity is resonant. The imaginary part of the relative effective permittivity determines the dissipation of the electromagnetic wave in the material. Thus the absorption is the maximal when the frequency is located in the resonant frequency.

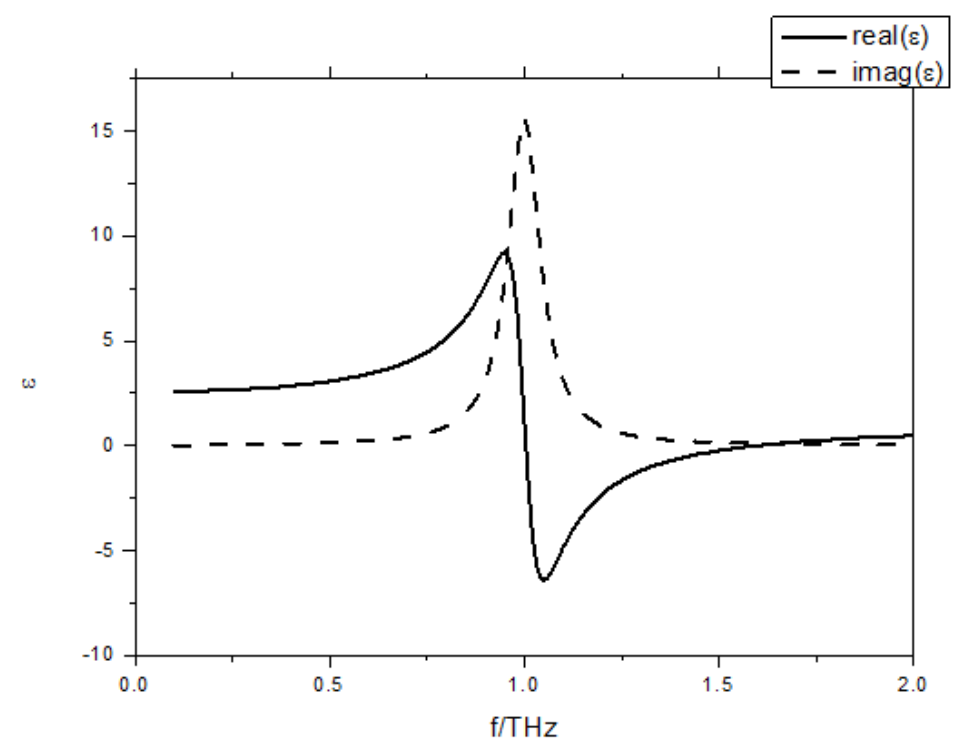

Figure 1. The relations between the real part and the imaginary part of the effective relative permittivity with the frequency.

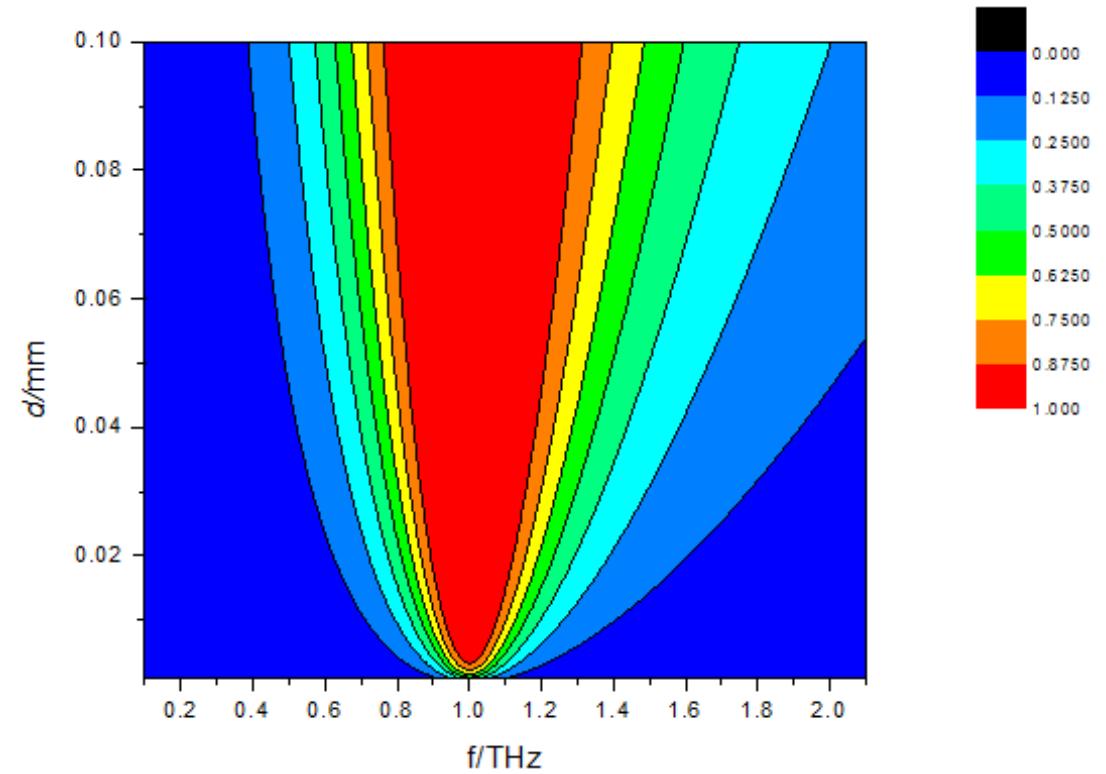

Figure 2. (color on line) The relations between the absorption with the frequency and the thickness.

Figure 2 is the result for the absorption of a single layer isotropic metamaterial with the frequency and the thickness of the metamaterial. The result shows that the frequency has a resonant peak and the bandwidth is determined by the thickness. As the increasing of the thickness, the bandwidth is bigger. These results mean that we can use a single layer of metamaterial to be the electromagnetic wave filter with the adjustable bandwidth.

For the multilayer system with the metamaterial, we can study the effect of the multilayer reflection to the absorption of the electromagnetic wave. Considering such a sandwiched three layers system, a layer of metamaterial is placed in two traditional material with the relative permittivity and the peameability as $\varepsilon_{r}=2.0, \mu_{r}=1.0$. The thickness of each 
layer is taken as $0.01 \mathrm{~mm}$. The absorption of the electromagnetic wave through such a three layer system is shown in Figure 3 as the dashed line. The absorption of the electromagnetic wave through such a single layer system with the thickness $0.01 \mathrm{~mm}$ is also shown in Figure 3 as the solid line for comparison. The results show that the absorption of the three layer system is bigger than the single layer system although that the thickness of the metamaterial for the two kind of the structure is the same. This is because that the interface multi-reflection of the multilayer system enlarger the absorption. We also plot the result for a periodic multilayer structure with the layer number as $N=21$ as dotted line in Figure 3. The thickness of each layer for a 21 layer system is taken as $0.01 \mathrm{~mm}$. The parameter of the traditional material is the same as the three layer system. From the plot we find that the bandwidth for a 21 layer number structure is bigger the three layer structure. It means that the bandwidth of the absorption is determined by the layer number.

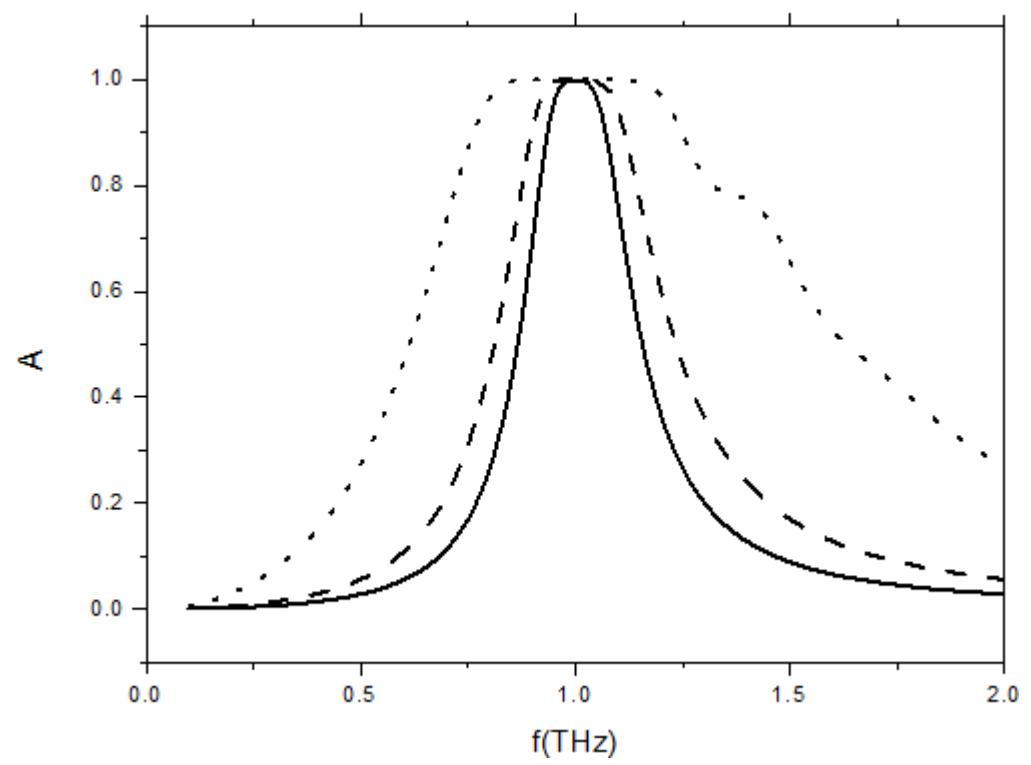

Figure 3. The relations between the absorption with the frequency for the single layer structure (solid line), the three layer structure (dashed line) and the multi-layer structure (dotted line) containing the isotropic metamaterials.

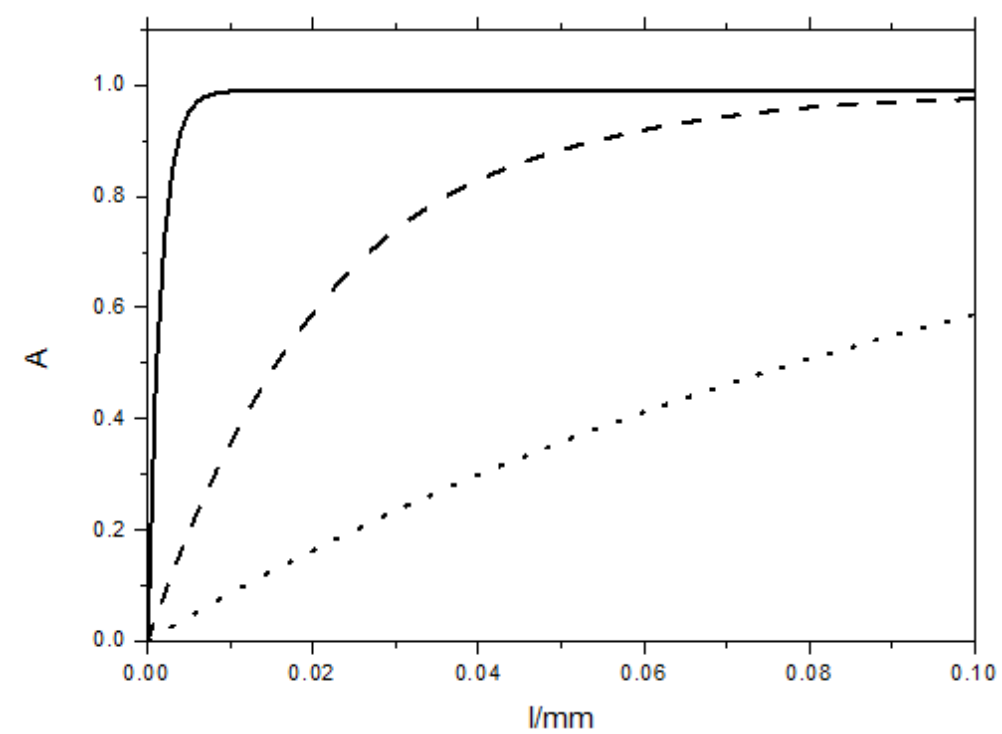

Figure 4. The relations between the absorption with the thickness of the metamaterial for a single layer structure with the frequency as $f=1.0 T H z$ (solid line), $f=1.2 \mathrm{THz}$ (dashed line) and $f=1.5 \mathrm{THz}$ (dotted line).

We also study the relation between the absorption with the thickness of the isotropic metamaterial for a single layer system which is shown in Figure 4. The plot give three different cases with the frequency $\mathrm{f}=1.0 \mathrm{THz}$ (solid line), $\mathrm{f}=1.2 \mathrm{THz}$ (dashed line) and $\mathrm{f}=1.5 \mathrm{THz}$ (dotted line). The absorption for the electromagnetic wave with the frequency
$1.0 \mathrm{THz}$ which is the resonant frequency is easier to achieve saturation.

For the anisotropic metamaterial, we study the absorption of the electromagnetic wave in different polarization through the single layer and multilayer structure with the anisotropic metamaterial. The following parameters 


$$
\begin{gathered}
\mu_{x}(\omega)=\mu_{z}(\omega)=1-\frac{\omega_{p, m}^{2}}{\omega^{2}+i \gamma_{m} \omega} \\
\mu_{y}(\omega)=10-\frac{\omega_{p, m}^{2}}{\omega^{2}+i \gamma_{m} \omega}
\end{gathered}
$$

are taken for anisotropic material. $\varepsilon_{x}=\varepsilon_{y}=\varepsilon_{z}=1$, $\omega_{p, m}=2 \pi \times 1.25 \mathrm{THz}$, and $\gamma_{m}=2 \pi \times 0.1 \mathrm{THz}$ are used in the calculation. The results for the absorptions As and Ap are shown in Figure 5 as solid line and dotted line respectively. The thickness is taken as $0.01 \mathrm{~mm}$ in the calculation. The absorption Ap achieves the maximum value near about $0.4 \mathrm{THz}$. However the absorption achieves the maximum value near about $1.3 \mathrm{THz}$. This is caused by the different permeability. The absorption is dependent on the polarization direction of the incident electromagnetic wave for the anisotropic metamaterial. Thus we can use the anisotropic metamaterial to be the polarization tunable absorber.

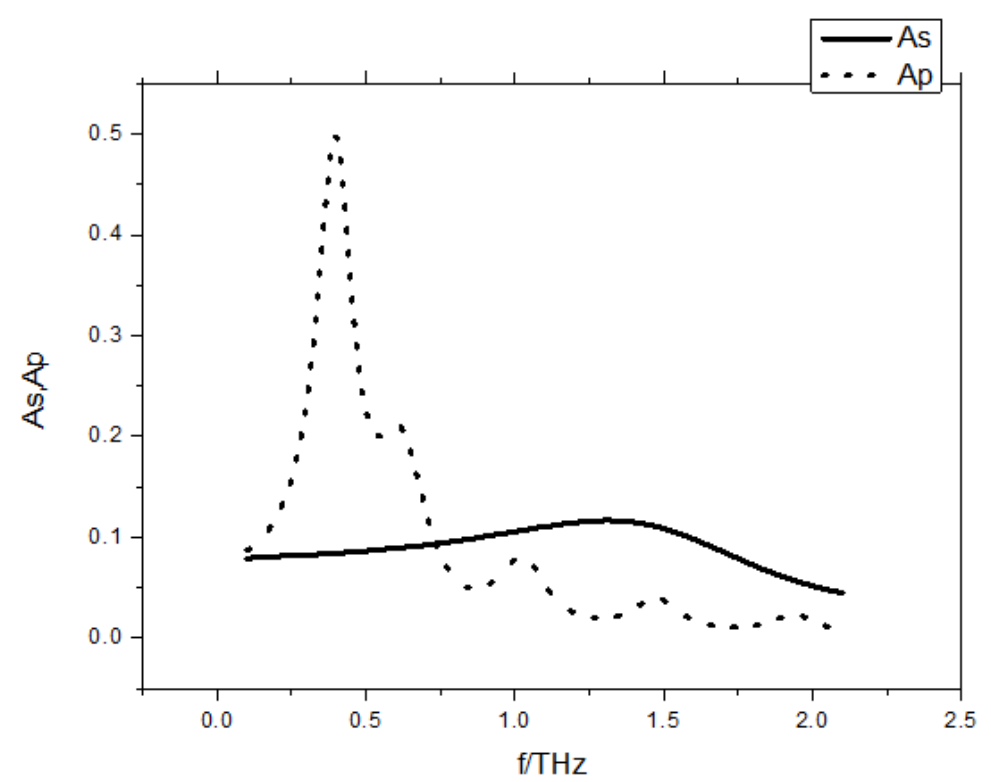

Figure 5. The relations between the absorption for the anisotropic metamaterial with the frequency for electromagnetic wave in the s polarization (solid line) and p polarization (dotted line).

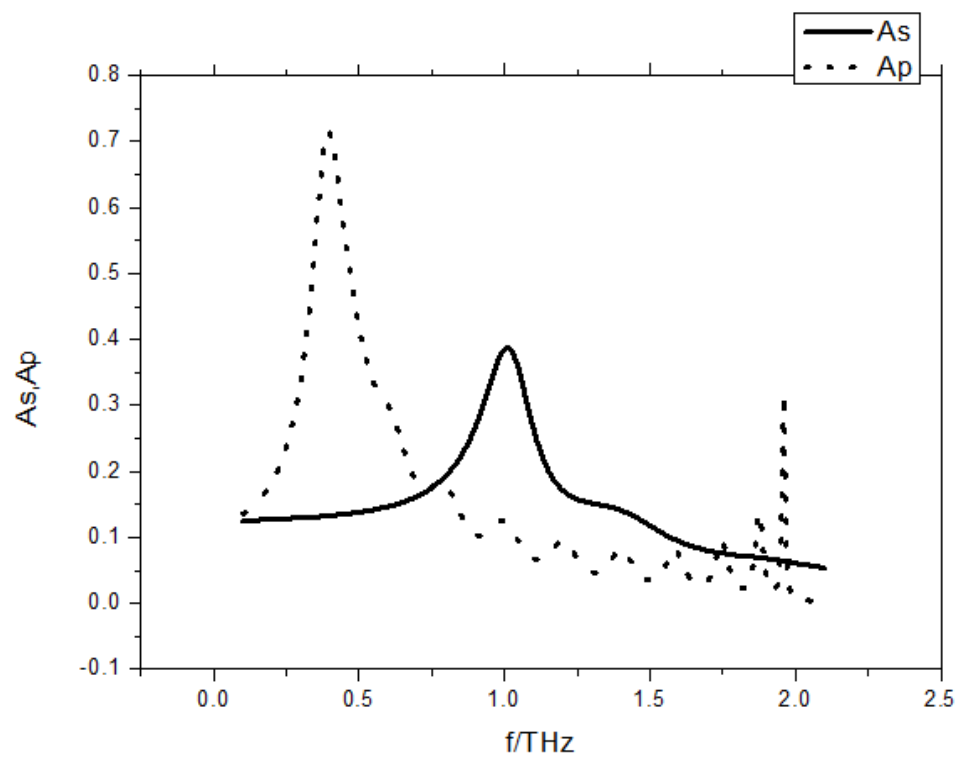

Figure 6. The relations between the absorption for the multilayer anisotropic metamaterial $(N=21)$ with the frequency for electromagnetic wave in the $s$ polarization (solid line) and p polarization (dotted line).

For the multilayer system with the anisotropic metamaterial, we taken the layer number $N=21$ shown in Figure 6 . Considering the multilayer system like this, a layer of metamaterial is separated by two traditional material with the relative permittivity and the peameability as $\varepsilon_{r}=2.0, \mu_{r}=1.0$. The thickness of the traditional material each layer is taken as $0.01 \mathrm{~mm}$ and the same as the anisotropic metamaterial. From the result we find that the absorption for the multilayer system 
is greater than the single layer system. At the same time the frequency for the maximum absorption is moving. So the layer number can also determine the frequency for the maximum absorption.

\section{Conclusion}

The absorption properties of the one dimensional structure with the metamaterial are calculated basing on the transfer matrix method. The absorption is approaching the maximum value, $100 \%$ when the freuquency of the incident wave is near the resonant frequency for the single layer isotropic metamaterials. The bandwidth of the incident wave becomes large when the thickness increases. This means that the single layer metamaterials can be used as the electromagnetic wave narrow band absorber and the bandwidth can be controlled by the thickness of the metamaterials. The interface effect makes the absorption larger for the three layer structure with the metamaterial and the traditional material. The reason is that increasing the number of the layer make the thickness of the structure larger, thus the bandwidth of the absorption is bigger. For the system with the anisotropic metamaterial, we study the absorption of the electromagnetic wave in different polarization. The absorption is different for different polarization which is caused by the different permeability. The absorption is dependent on the polarization direction of the incident electromagnetic wave for the anisotropic metamaterial. Thus we can use the anisotropic metamaterial to be the polarization tunable absorber. The layer number can also determine the frequency for the maximum absorption. Increasing the layer number the absorption changes with the frequency.

\section{Acknowledgements}

This work was supported by the Fundamental Research Funds for the Central Universities under Grant No. $2015 \mathrm{MS} 01$.

\section{References}

[1] D. R. Smith, W. J. Padilla, D. C. Vier, S. C. Nemat-Nasser, and S. Schultz, Composite Medium with Simultaneously Negative Permeability and Permittivity, Phys. Rev. Lett. 844184 (2000)

[2] L. F. Shen, S. L. He and S. S. Xiao, Stability and quality factor of a one-dimensional subwavelength cavity resonator containing a left-handed material, Phys. Rev. B 69115111 (2004)

[3] J. Li, L. Zhou, C. T. Chan, P. Sheng, Photonic Band Gap from a Stack of Positive and Negative Index Materials, Phys. Rev. Lett. 90083901 (2003)

[4] D. R. Smith and D. Schurig, Electromagnetic Wave Propagation in Media with Indefinite Permittivity and Permeability Tensors, Phys. Rev. Lett. 90077405 (2003)
[5] L. Zhou, C. T. Chan, and P. Sheng, Anisotropy and oblique total transmission at a planar negative-index interface, Phys. Rev. B $68115424(2003)$

[6] S. Sun, X. Huang, and L. Zhou, Two-dimensional complete photonic gaps from layered periodic structures containing anisotropic left-handed metamaterials, Phys. Rev. E 75066602 (2007)

[7] L. Hu and S. T. Chui, Characteristics of electromagnetic wave propagation in uniaxially anisotropic left-handed materials, Phys. Rev. B 66, 085108 (2002)

[8] Q. Cheng and T. J. Cui, Electromagnetic interactions between a line source and anisotropic biaxial media with partially negative constitutive parameters, J. of Appl. Phys. 98, 074903 (2005)

[9] J. Hao and L. Zhou, Electromagnetic wave scatterings by anisotropic metamaterials: Generalized $4 \times 4$ transfer-matrix method, Phys. Rev. B 77094201 (2008)

[10] J. Hao, M. Qiu and L. Zhou, Manipulate light polarizations with metamaterials: From microwave to visible, Front. Phys. 5(3), 291 (2010)

[11] H. F. Ma, W. X. Tang, Q. Cheng and T. J. Cui, A single metamaterial plate as bandpass filter, transparent wall, and polarization converter controlled by polarizations Appl. Phys. Lett. 105081908 (2014)

[12] Y. Dong, X. Zhang, Quantum-optical input-output relations and entanglement distillation by anisotropic planar multilayers, J. Opt. 13035401 (2011)

[13] Y. Dong, C. Liu, Electromagnetic field quantization and input-output relation for anisotropic magnetodielectric metamaterial, Chin. Phys. B 24064206 (2015)

[14] Y. Dong, J. You, Propagation of polarized photons through a cavity with an anisotropic metamaterial, Front. Phys. 11, 114206 (2016)

[15] N. I. Landy, S. Sajuyigbe, J. J. Mock, D. R. Smith, W. J. Padilla, Perfect Metamaterial Absorber, Phys. Rev. Lett. 100, 207402 (2008)

[16] C. M. Watts, X. L. Liu, W. J. Padilla, Metamaterial Electromagnetic Wave Absorbers, Advanced Materials, 24 (23): OP98 (2012)

[17] H. Tao et al., "A metamaterial absorber for the terahertz regime: Design, fabrication and characterization," Opt. Exp., vol. 16, no. 10 , pp. 7181-7188 (2008)

[18] X. Liu, T. Starr, A. F. Starr, and W. J. Padilla, "Infrared spatial and frequency selective metamaterial with near-unity absorbance,” Phys. Rev. Lett., vol. 104, 207403 (2010)

[19] L. Huang et al., Experimental demonstration of terahertz metamaterial absorbers with a broad and flat high absorption band, Opt. Lett., vol. 37, no. 2, pp. 154-156 (2012)

[20] J. Grant, Y. Ma, S. Saha, A. Khalid, and D. R. S. Cumming, "Polarization insensitive, broadband terahertz metamaterial absorber," Opt. Lett., vol. 36, no. 17, pp. 3476-3478 (2011) 